\title{
WEAKLY NORMAL FILTERS AND IRREGULAR ULTRAFILTERS
}

\author{
BY
}

\section{A. KANAMORI}

ABSTRACT. For a filter over a regular cardinal, least functions and the consequent notion of weak normality are described. The following two results, which make a basic connection between the existence of least functions and irregularity of ultrafilters, are then proved: Let $U$ be a uniform ultrafilter over a regular cardinal $\kappa$. (a) If $\kappa=\lambda^{+}$, then $U$ is not $\left(\lambda, \lambda^{+}\right)$-regular iff $U$ has a least function $f$ such that $\left\{\xi<\lambda^{+} \mid \operatorname{cf}(f(\xi))=\lambda\right\} \in U$. (b) If $\omega<\mu<\kappa$ and $U$ is not $(\omega, \mu)$-regular, then $U$ has a least function.

In this paper is considered a relatively new class of filters, those which satisfy a property abstracted from normal ultrafilters over a measurable cardinal. The first section discusses these filters in a general context, and the second shows their relevance to the study of the regularity of ultrafilters. The set theoretical notation and terminology is standard, in particular $\alpha, \beta, \gamma, \ldots$ are variables for ordinals while $\kappa, \lambda, \mu, \ldots$ are reserved for cardinals. In fact, $\kappa$ will denote an arbitrary but fixed regular cardinal throughout the discussion. It is always assumed that a filter over $\kappa$ is proper and contains the sets $\{\xi \mid \alpha<\xi<\kappa\}$ for every $\alpha<\kappa$, so that ultrafilters are always uniform.

This material forms part of the third chapter of the author's doctoral dissertation [4], but the results (except 2.5) were obtained some time ago in 1974.

1. Weakly normal filters. A series of easy definitions culminate in the main concept; recall that a set $X$ has positive measure with respect to a filter $F$ iff $X$ meets every element of $F$.

1.1. Definitions. Let $F$ be a filter over $\kappa$.

(i) $f \in{ }^{\kappa} \kappa$ is unbounded $(\bmod F)$ iff $\{\xi<\kappa \mid \alpha<f(\xi)\}$ has positive measure for every $\alpha<\kappa$.

(ii) $f \in{ }^{\kappa} \kappa$ is almost 1-1 iff for every $\alpha<\kappa,\left|f^{-1}(\{\alpha\})\right|<\kappa . f \in \kappa^{K}$ is almost 1-1 $(\bmod F)$ iff there is a set $X$ of positive measure so that $f \mid X$ is almost $1-1$, i.e. for every $\alpha<\kappa,\left|f^{-1}(\{\alpha\}) \cap X\right|<\kappa$.

(iii) $F$ is a $p$-point filter iff every function unbounded $(\bmod F)$ is almost 1-1 $(\bmod F)$.

(iv) $f \in{ }^{\kappa} \kappa$ is a least function $(\bmod F)$ iff $f$ is unbounded $(\bmod F)$ yet

Received by the editors May 7, 1975 and, in revised form, June 2, 1975.

AMS (MOS) subject classifications (1970). Primary 04A10, $02 \mathrm{K35}$. ultrafilters.

Key words and phrases. Least functions, weakly normal filters, regularity of uniform 
$\{\xi<\kappa \mid g(\xi)<f(\xi)\} \in F$ implies that $g$ is not unbounded $(\bmod F)$.

(v) $F$ is a weakly normal filter iff any function $\in^{{ }^{K}}{ }$ regressive on a set in $F$ is not unbounded $(\bmod F)$, i.e. the identity function $\in^{\kappa} K$ is a least function $(\bmod F)$.

When $k=\omega$ and $F$ is an ultrafilter, then 1.1(iii) coincides with the usual notion of a $p$-point in $\beta N$, the Stone-Čech compactification of the integers. Notice that if there is a least function $f$ for $F$ and $F$ is ultra, then $f_{*}(F)=$ $\left\{X \subseteq \kappa \mid f^{-1}(X) \in F\right\}$ is weakly normal. Weak normality is typical of several fruitful concepts that have developed through the study of large cardinal axioms; the existence of countably incomplete ultrafilters with least functions may still be possible even on $\omega_{1}$.

The following characterizations are easy to establish. Let $C_{\lambda}$ where $\lambda$ is a regular cardinal denote the filter generated by the closed unbounded subsets of $\lambda$.

1.2. Proposition. For any filter $F$ over $k$, the following are equivalent:

(i) $F$ is weakly normal.

(ii) Every filter extension of $F$ is weakly normal.

(iii) If $X_{\alpha}$ for $\alpha<\kappa$ are sets of positive measure such that $\alpha<\beta \rightarrow X_{\beta}$ $\subseteq X_{\alpha}$, then $\left\{\beta<\kappa \mid \alpha<\beta \rightarrow \beta \in X_{\alpha}\right\}$ has positive measure.

(iv) $F$ is a p-point filter extending $C_{\kappa}$.

Proof. (i) $\rightarrow$ (ii) is obvious.

(ii) $\rightarrow$ (iii). Let $G$ be the weakly normal filter generated by $F \cup\left\{X_{\alpha} \mid \alpha<\right.$ $\kappa\}$. If the set in question does not have positive $F$ measure, then $\{\beta<k \mid$ for some $\left.f(\beta)<\beta, \beta \notin X_{f(\beta)}\right\} \in G$. But then $f$ is bounded $(\bmod G)$ and the $X_{\alpha}$ 's are descending, so that for some $\delta<\kappa, X_{\delta} \notin G$, which is a contradiction.

(iii) $\rightarrow$ (iv). To show that $F$ is a $p$-point, if $f$ is unbounded $(\bmod F)$, let $X_{\alpha}=\{\xi \mid \alpha<f(\xi)\}$ in (iii). If $C_{\kappa} \nsubseteq F$, there is a $Y \subseteq \kappa$ of positive measure whose complement is closed unbounded in $\kappa$. As usual, $f \in{ }^{\kappa} \kappa$ defined by $f(\beta)=$ $\sup (\beta \cap(\kappa-Y))$ is an almost 1-1 function regressive on $Y$. The sets $X_{\alpha}=$ $\{\beta \in Y \mid \alpha<f(\beta)\}$ for $\alpha<\kappa$ are descending, so that by (iii), $\{\beta<\kappa \mid \alpha<\beta \rightarrow$ $\left.\beta \in X_{\alpha}\right\}$ has positive measure. But for such $\beta, \beta \leqslant f(\beta)$, a contradiction.

(iv) $\rightarrow$ (i). Suppose $f \in{ }^{\kappa} \kappa$ is regressive. If for every $\alpha<\kappa, X_{\alpha}=\{\beta<$ $\kappa \mid f(\beta)>\alpha\}$ has positive measure, since $F$ is a $p$-point, there is a set $Y$ of positive measure such that $f$ is almost $1-1$ on $Y$. But since $Y$ is stationary, this contradicts a well-known result of Fodor, as $f$ must be constant on a stationary subset of $Y$.

Weak normality is a different concept from normality in the sense of Solovay [9], since in particular no completeness property for filters need be hypothesized. However, the two concepts are closely related if the filter is K-complete: 
1.3. Proposition. For any $k$-complete filter $F$ over $k$, the following are equivalent:

(i) $F$ is weakly normal.

(ii) $F$ is a normal $k$-saturated filter (in the sense of Solovay [9]).

Proof. If $F$ is weakly normal and $f$ is regressive on a set of positive measure, then for some $\alpha<\kappa,\{\xi \mid f(\xi)<\alpha\}$ has positive measure. By $\kappa$-completeness, it follows that $f$ is constant on a set of positive measure. Suppose now that $F$ is not $k$-saturated. Let $\left\langle X_{\alpha} \mid \alpha<k\right\rangle$ be a partition of $k$ such that for $\alpha<$ $\kappa, X_{\alpha}$ has positive measure. We can assume that $\beta \in X_{\alpha}$ implies $\beta>\alpha$ for $\alpha$ such that $0<\alpha<\kappa$ by throwing all contrary ordinals into $X_{0}$. But then, $f$ defined by $f(\beta)=\alpha$ iff $\beta \in X_{\alpha}$ is regressive but must be unbounded $(\bmod F)$, a contradiction.

Conversely, if $f$ is regressive, let $T=\left\{\alpha<k \mid f^{-1}(\{\alpha\})\right.$ has positive measure $\}$. By $\kappa$-saturation, $|T|<\kappa$. So, if $\delta=\sup T,\{\beta<\kappa \mid f(\beta) \leqslant \delta\} \in F$ : otherwise, $\{\beta<\kappa \mid \delta<f(\beta)<\beta\}$ would have positive measure, and by normality there would be a $\rho>\delta$ so that $f^{-1}(\{\rho\})$ has positive measure, contradicting the definition of $\delta$.

The following well-known definition is due to Keisler.

1.4. Definition. If $\mu \leqslant \lambda$, an ultrafilter $U$ is $(\mu, \lambda)$-regular iff there are $\lambda$ sets in $U$ so that any $\mu$ of them has empty intersection.

Keisler first considered regularity as a measure of width in the context of model theory. Roughly, he showed that regularity assumptions on an ultrafilter result in their ultraproducts having large cardinality (see Chang-Keisler [2] for details). The following result is due to Ketonen.

1.5. THEOREM (KeTONEN [6]). Suppose an ultrafilter $U$ over $k$ has a least function $f$. Then $U$ is $(\mu, \kappa)$-regular iff $\{\alpha \mid \operatorname{cf}(f(\alpha))<\mu\} \in U$.

Note that any ultrafilter which has a least function cannot be $(\omega, \kappa)$-regular. When $k$ is weakly inaccessible and $U$ over $k$ is weakly normal, it follows from the theorem that either: (i) $\{\alpha<\kappa \mid \alpha$ is regular $\} \in U$ and $U$ is not $(\mu, \kappa)$-regular for any $\mu<\kappa$, or (ii) for some $\mu<\kappa,\{\alpha \mid \operatorname{cf}(\alpha)<\mu\} \in U$ and $U$ is $(\mu, \kappa)$-regular. Hence, by a well-known result of Solovay [9], we get a result of Silver in a strong form:

1.6. THEOREM. Any ultrafilter extension of a normal $k$-complete $k$-saturated filter over $k$ is a weakly normal ultrafilter which is not $(\mu, \kappa)$-regular for any $\mu<\kappa$.

In contrast, consider the following observation:

1.7. Proposition. Let $F$ be a filter over $k$ such that there exists a parti- 
tion $\left\langle X_{\alpha} \mid \alpha<\kappa\right\rangle$ of $\kappa$ with each $X_{\alpha}$ of positive measure. Then there is an extension of $F$ which is $(\omega, \kappa)$-regular.

Proof. Let $f: \kappa \leftrightarrow[\kappa]<\omega$ be bijective and consider for $\beta<\kappa$ the sets $Y_{\beta}=\bigcup\left\{X_{\alpha} \mid \beta \in f(\alpha)\right\}$. They satisfy the finite intersection property, yet any intersection of infinitely many of them is empty. Hence, the filter generated by $F \cup\left\{Y_{\beta} \mid \beta<\kappa\right\}$ is as required.

Suppose now that $\kappa$ is a measurable sardinal, $\mu<\kappa$, and $\left\{N_{\alpha} \mid \alpha<\mu\right\}$ is a

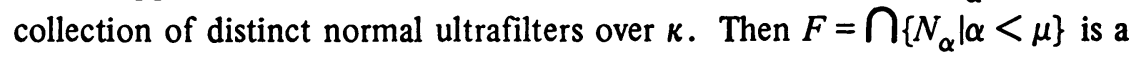
$\kappa$-complete weakly normal filter and hence $\kappa$-saturated. In fact, it is not hard to see that $F$ is exactly $\mu^{+}$-saturated. Moreover, if $\left\langle X_{\alpha} \mid \alpha<\mu\right\rangle$ is a partition of $\kappa$ such that $X_{\alpha} \in N_{\alpha}$, then $F \cup\left\{X_{\alpha}\right\}$ must generate $N_{\alpha}$ : if $\alpha<\mu$ and $Y \subseteq X_{\alpha}$ so that $Y \notin N_{\alpha}$, then $\kappa-Y \in F$, so $Y$ does not have positive measure. However, in $L[U]$ where $U$ is a normal ultrafilter over a measurable cardinal $\kappa$, it is the case that the only normal $k$-complete $\kappa^{+}$-saturated filter over $\kappa$ is $U \cap L[U]$ itself (see Kunen [7]).

1.8. Concerning forcing and weakly normal filters, note that if $F$ is weakly normal over $\kappa$ such that $\{\alpha \mid \mathrm{cf}(\alpha) \geqslant \mu\} \in F$, then in any $\mu$-c.c. extension of the universe $F$ generates a weakly normal filter $F^{\prime}$, by an idea in Ketonen [6]:

If $0<\alpha<\kappa$ implies $f(\alpha)<\alpha$ with Boolean value one in the extended universe, by $\mu$-c.c. there is a standard function $g$ so that for every $\alpha<\kappa, f(\alpha) \leqslant$ $g(\alpha)$ and $g(\alpha)$ is the supremum of less than $\mu$ ordinals each less than $\alpha$. Hence, $\{\alpha<\kappa \mid g(\alpha)<\alpha\} \in F$ and $f$ will be bounded $\left(\bmod F^{\prime}\right)$, as $g$ is bounded $(\bmod F)$ in the standard universe.

Thus, one can freely reduce the complexity of $F$ by collapsing the cardinal $\mu$, for instance. As another observation, Kunen's concept of a huge cardinal (i.e. a cardinal $\kappa$ such that there is an elementary embedding $j: V \rightarrow M$ of the universe into a transitive submodel $M$ which first moves $k$, where $M$ is closed under sequences of length $j(k)$ ) yields a $k$-complete, weakly normal, uniform ultrafilter $U$ over $j(\kappa)$ such that $\{\alpha<j(\kappa) \mid \operatorname{cf}(\alpha)=\kappa\} \in U$ (see Ketonen [6]). By collapsing $k$ to $\omega_{1}$, one can get a weakly normal filter over an inaccessible cardinal $\lambda$ any of whose ultrafilter extensions will be $\left(\omega_{2}, \lambda\right)$-regular but not $\left(\omega_{1}\right.$, $\lambda)$-regular.

2. Irregular ultrafilters. The following rather easy theorem, proved two years ago, first connected irregularity phenomena to the existence of least functions, and led to the deeper results of Jensen [3] and Ketonen. The expression $[f]$ denotes the usual equivalence class of $f$ modulo the ultrafilter concerned.

2.1. THEOREM. If an ultrafilter $U$ over $\kappa$ is not $(\omega, \kappa)$-regular, then there is a least almost $1-1$ function $(\bmod U)$. 
Proof. Argue by contradiction, and inductively define a sequence $\left\langle f_{\alpha}\right|$ $\alpha<\kappa\rangle$ of almost 1-1 functions, with the property that $\alpha<\beta<\kappa$ implies $f_{\beta}(\xi) \leqslant$ $f_{\alpha}(\xi)$ for every $\xi<\kappa$, as follows:

Suppose $\delta<\kappa$ and $f_{\alpha}$ for $\alpha<\delta$ have been defined. If $\delta=\beta+1$, let $f_{\delta}$ be any almost $1-1$ function such that $\left[f_{\delta}\right]<\left[f_{\beta}\right]$ and $f_{\delta}(\xi) \leqslant f_{\beta}(\xi)$ for every $\xi<\kappa$. If $\delta$ is a limit ordinal, define $f_{\delta}$ by $f_{\delta}(\xi)=\min \left\{f_{\alpha}(\xi) \mid \alpha<\delta\right\}$. Note that $f_{\delta}$ is almost $1-1$.

To complete the proof, set for $\alpha<\kappa, X_{\alpha}=\left\{\xi \mid f_{\alpha}(\xi)>f_{\alpha+1}(\xi)\right\} \in U$. If any infinite subcollection of the $X_{\alpha}$ 's had a nonempty intersection, then there would be an infinite descending sequence of ordinals. Hence, $U$ is $(\omega, \kappa)$-regular.

Notice that if the $U$ of the theorem were also a $p$-point, then it would have a least function. There is one known irregularity hypothesis for which this is true:

2.2. Proposition (Ketonen [1]). If an ultrafilter $U$ over $\lambda^{+}$is not $\left(\lambda, \lambda^{+}\right)$-regular, then $U$ is a p-point; moreover, if $f: \lambda^{+} \rightarrow \lambda^{+}$is unbounded $(\bmod U)$, then there is an $X \in U$ so that for every $\alpha<\lambda^{+},\left|X \cap f^{-1}(\{\alpha\})\right|<\lambda$.

The following characterization was announced in Kanamori [5].

2.3. THEOREM. For an ultrafilter $U$ over $\lambda^{+}, U$ is not $\left(\lambda, \lambda^{+}\right)$-regular iff it has a least function $f$ so that $\left\{\alpha<\lambda^{+} \mid \operatorname{cf}(f(\alpha))=\lambda\right\} \in U$.

Proof. See 2.1, 2.2, and 1.5. Also, the following is a short proof of one direction.

Assume $f$ is a least function $(\bmod U)$ such that $\left\{\alpha<\lambda^{+} \mid \operatorname{cf}(f(\alpha))=\lambda\right\} \in U$. If $\left\{X_{\alpha} \mid \alpha<\lambda^{+}\right\}$is a collection of sets in $U$, define $g: \lambda^{+} \rightarrow \lambda^{+}$by $g(\alpha)=$ $\sup \left\{\beta<f(\alpha) \mid \alpha \in X_{\beta}\right\}$. If $[g]<[f]$, then $g$ is bounded by some $\delta<\lambda^{+}(\bmod U)$, and so $X_{\delta} \notin U$, which is contradictory. Thus, $[g]=[f]$, and $\left\{\alpha<\lambda^{+} \mid g(\alpha)=\right.$ $f(\alpha)$ and $\operatorname{cf}(f(\alpha))=\lambda\} \in U$. But any such $\alpha$ must be in at least $\lambda$ of the $X_{\beta}$ 's. Hence, $U$ is not $\left(\lambda, \lambda^{+}\right)$-regular.

2.4. COROLLARY. If $\lambda$ is singular then every uniform ultrafilter over $\lambda^{+}$ is $\left(\lambda, \lambda^{+}\right)$-regular.

It is still not known whether a $U$ as in the theorem can exist, but Ketonen has recently used the theorem to show that if there were such a $U$, then $0^{\#}$ exists. Also, a very weak form of Kurepa's Hypothesis called $w K H_{\omega_{1}}\left(C_{\omega_{1}}\right)$ which is known to refute Chang's Conjecture (see [1] for details) suffices, by the theorem, to show that every uniform ultrafilter over $\omega_{1}$ is $\left(\omega, \omega_{1}\right)$-regular.

By the forcing considerations of 1.8 , observe that if an ultrafilter $U$ over $\lambda^{+}$is a non- $\left(\lambda, \lambda^{+}\right)$-regular ultrafilter which we can now assume to be weakly 
normal, and if $\lambda$ is either a strongly inaccessible or a successor cardinal, then in the usual forcing extension by the $\lambda$-c.c. algebra which collapses every cardinal below $\lambda$ to $\omega, U$ generates a uniform, weakly normal filter over $\omega_{2}$ which contains the set $\left\{\alpha<\omega_{2} \mid \operatorname{cf}(\alpha)=\omega_{1}\right\}$. Thus, no extension of this filter can be $\left(\omega_{1}, \omega_{2}\right)$-regular. This last observation amplifies a fact known to Ketonen [6].

Recent work of Jensen and Ketonen sheds more light on irregularity of ultrafilters. Jensen [3] showed that if $\lambda$ is regular so that $2^{\lambda}=\lambda$ and there is a uniform weakly normal ultrafilter over $\lambda$, then $0^{\#}$ exists and $\lambda$ is ineffable in $L$. The following theorem is due independently to the author and Ketonen, although Ketonen first proved it some time before the author did. Ketonen apparently has much further amplifications which use close unbounded sets, but the theorem is presented here since the proof needs little further sophistication beyond 2.1 .

2.5. THEOREM (KETONEN, INDEPENDENTLY). Let $U$ be an ultrafilter over $\kappa$ which is not $(\omega, \kappa)$-regular. If $U$ has no least function, then there is a sequence of functions $\left\langle f_{\alpha}\right| \alpha\langle k\rangle \subseteq{ }^{k} \kappa$ and a sequence of ordinals $\left\langle\gamma_{\alpha}\right| \alpha\langle k\rangle \subseteq \kappa$ so that:

(i) each $f_{\alpha}$ is unbounded $(\bmod U)$;

(ii) if $\alpha<\beta<\kappa,\left[f_{\beta}\right]<\left[f_{\alpha}\right]$;

(iii) if $\alpha<\beta<\kappa$ and $\gamma_{\beta} \leqslant f_{\alpha}(\xi)$, then $f_{\beta}(\xi) \leqslant f_{\alpha}(\xi)$.

Proof. Proceed by induction. Suppose $\delta<k$ and $\left\langle f_{\alpha} \mid \alpha<\delta\right\rangle$ and $\left\langle\gamma_{\alpha}\right|$ $\alpha<\delta>$ have already been chosen. If $\delta=\beta+1$, let $\gamma_{\delta}=\gamma_{\beta}$ and $f_{\delta}$ any function unbounded $(\bmod U)$ such that $\left[f_{\delta}\right]<\left[f_{\beta}\right]$ and $f_{\delta}(\xi) \leqslant f_{\beta}(\xi)$ for every $\xi<\kappa$. If $\delta$ is a limit ordinal, we need the following:

Lemma. There is a $\rho<k$ so that whenever $\rho<\sigma<k,\{\xi<\kappa \mid(\alpha<\delta$ and $\left.\left.\rho \leqslant f_{\alpha}(\xi)\right) \rightarrow \sigma \leqslant f_{\alpha}(\xi)\right\} \in U$.

Proof. Argue by contradiction and inductively define an increasing sequence of ordinals $\left\langle\rho_{\zeta} \mid \zeta<\kappa\right\rangle$ by:

$\rho_{0}=\sup \left\{\gamma_{\alpha} \mid \alpha<\delta\right\}$,

$\rho_{\eta}=\sup \left\{\rho_{\zeta} \mid \zeta<\eta\right\}, \eta$ a limit, and

$\rho_{\xi+1}=$ least $\sigma>\rho_{\zeta}$ so that $\left\{\xi \mid \rho_{\xi} \leqslant f_{\alpha}(\xi)<\sigma\right.$ for some $\left.\alpha<\delta\right\} \in U$. But then, if $Y_{\xi}=\left\{\xi \mid \rho_{\xi} \leqslant f_{\alpha}(\xi)<\rho_{\xi+1}\right.$ for some $\left.\alpha<\delta\right\}$ for $\zeta<\kappa,\left\{Y_{\xi} \mid \xi<\kappa\right\}$ $\subseteq U$ shows that $U$ is $(\omega, \kappa)$-regular, else there would be a $\xi<\kappa$ and an infinite set of ordinals $\left\{\alpha_{n} \mid n \in \omega\right\}$ so that:

$$
\rho_{0} \leqslant f_{\alpha_{0}}(\xi)<f_{\alpha_{1}}(\xi)<f_{\alpha_{2}}(\xi) \ldots,
$$

but then $\alpha_{0}>\alpha_{1}>\alpha_{2} \cdots$. This contradiction establishes the Lemma.

To continue the proof of the theorem, let $\gamma_{\delta}$ be a $\rho$ as in the Lemma, 
where we can suppose $\gamma_{\delta}>\sup \left\{\gamma_{\alpha} \mid \alpha<\delta\right\}$. Now define $f_{\delta}$ by

$$
f_{\delta}(\xi)=\left\{\begin{array}{l}
0 \text { if } f_{\alpha}(\xi)<\gamma_{\delta} \text { for every } \alpha<\delta, \\
\min \left\{f_{\alpha}(\xi) \mid f_{\alpha}(\xi) \geqslant \gamma_{\delta}\right\} \text { otherwise. }
\end{array}\right.
$$

Since $\left\{\xi \mid f_{\delta}(\xi) \neq 0\right\} \in U$, if $f_{\delta}$ were bounded $(\bmod U)$, then for some $\sigma<\kappa$, $\left\{\xi \mid \gamma_{\delta} \leqslant f_{\alpha}(\xi)<\sigma\right.$ for some $\left.\alpha<\delta\right\} \in U$, which contradicts the choice of $\gamma_{\delta}$. The proof is now complete.

2.6. CoRollary. If $\omega \leqslant \lambda<\kappa$ and an ultrafilter $U$ over $\kappa$ is not ( $\omega$, $\lambda)$-regular, then $U$ has a least function.

PROof. Let $\gamma=\sup \left\{\gamma_{\alpha} \mid \alpha<\lambda\right\}<\kappa$, and for $\alpha<\lambda$, set $X_{\alpha}=\left\{\xi \mid f_{\alpha}(\xi)>\right.$ $\left.f_{\alpha+1}(\xi)>\gamma\right\} \in U$. As before, the existence of the $X_{\alpha}$ 's shows that $U$ is $(\omega, \lambda)$ regular, else we get an infinite descending sequence of ordinals.

It follows easily from this corollary and previous remarks that in $L$, every uniform ultrafilter over a regular cardinal $\kappa$ is $(\omega, \lambda)$-regular for every $\lambda<\kappa$. Hardly anything is known about uniform ultrafilters over singular cardinals, in $L$ or elsewhere. Finally, note that the corollary can be combined with results in [3] to show that if $\lambda<k$ are regular cardinals and there is a uniform ultrafilter over $k$ which is $\lambda$-descendingly complete (see Prikry [8] for details on this concept), then $0^{\#}$ exists, a result also known to Koppelberg.

\section{REFERENCES}

1. M. Benda and J. Ketonen, Regularity of ultrafilters, Israel J. Math. 17 (1974), $231-240$.

2. C. C. Chang and H. J. Keisler, Model theory, North-Holland, Amsterdam, 1973.

3. R. Jensen and B. Koppelberg, A note on ultrafilters; R. Jensen, Addendum to 'A note on ultrafilters' (both unpublished).

4. A. Kanamori, Ultrafilters over uncountable cardinals, Doctoral Dissertation, Univ. of Cambridge, 1975.

5. - A characterization of nonregular ultrafilters, Notices Amer. Math. Soc. 21 (1974), A-381. Abstract \#74T-E45.

6. J. Ketonen, Strong compactness and other cardinal sins, Ann. Math. Logic 5 (1972), 47-76.

7. K. Kunen, Some applications of iterated ultrapowers in set theory, Ann. Math. Logic 1 (1970), 179-227. MR 43 \#3080.

8. K. Prikry, On descendingly complete ultrafilters, Cambridge Summer School in Mathematical Logic, Lecture Notes in Math., vol. 337, Springer-Verlag, New York, 1973, pp. 459-488.

9. R. M. Solovay, Real-valued measurable cardinals, Proc. Sympos. Pure Math., vol. 13, part 1, Amer. Math. Soc., Providence, R. I., 1971, pp. 397-428. MR 45 \#55.

\section{DEPARTMENT OF
CAMBRIDGE, ENGLAND}

Current address: Department of Mathematics, University of California, Berkeley, California 94720 